\title{
optica
}

\section{Hybrid-cascaded generation of tripartite telecom photons using an atomic ensemble and a nonlinear waveguide: supplementary material}

\author{
Dong-Sheng Ding, ${ }^{1,2,+}$ WeI ZhANG, ${ }^{1,2,+}$ ShuaI ShI, ${ }^{1,2,+}$ ZHI-YUAN ZHOU, ${ }^{1,2}$ \\ YAN LI, ${ }^{1,2}$ BAO-SEN SHI, ${ }^{1,2, *}$ AND GuANG-CAN GUO ${ }^{1,2}$ \\ ${ }^{1}$ Key Laboratory of Quantum Information, University of Science and Technology of China, Hefei, Anhui 230026, China \\ ${ }^{2}$ Synergetic Innovation Center of Quantum Information \& Quantum Physics, University of Science and Technology of China, Hefei, Anhui \\ 230026, China \\ *Corresponding author: drshi@ustc.edu.cn ${ }^{+}$These authors contributed to this article equally
}

Published 10 July 2015

This document provides supplementary information to "Hybrid-cascaded generation of tripartite telecom photons using an atomic ensemble and a nonlinear waveguide," http://dx.doi.org/10.1364/optica.2.000642.

(C) 2015 Optical Society of America

http://dx.doi.org/10.1364/optica.2.000642.s001

Coincidence window shift with detuning of pump laser 1

The pump laser with the wavelength of $795 \mathrm{~nm}$ is stabilized using the spectrum locking technique. The laser is red-detuned by $1.45 \mathrm{GHz}$ with the ${ }^{87} \mathrm{Rb}$ atomic transition of $5 \mathrm{~S}_{1 / 2}(\mathrm{~F}=1)$ $>5 \mathrm{P}_{1 / 2}\left(\mathrm{~F}^{\prime}=1\right)$ using a $1.5 \mathrm{GHz}$ acousto-optic modulator. In our experiment, if we change the detuning of the 795-nm laser, then the time-correlation window would shift because of the delay of the 780-nm photon, as shown in Fig. 1S. The reason for this is that the linear susceptibility of the generated $780-\mathrm{nm}$ photon is modulated by the detuning of 795-nm laser; please refer to [1] for a more detailed description of this FWM delay effect. We therefore need to stabilize the 795-nm laser for appropriate experimental performance over long periods.

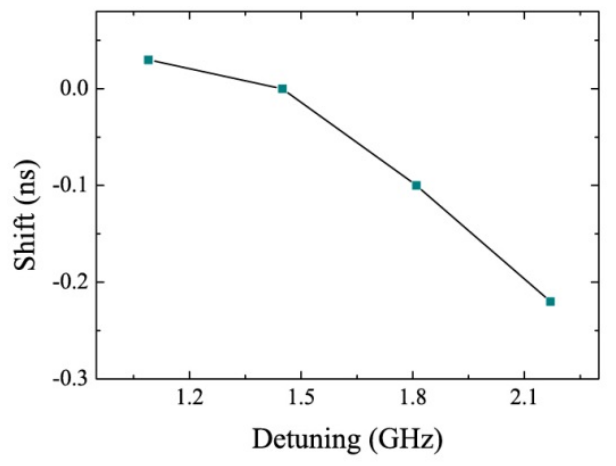

Fig. S1. The coincidence window is shifted by modulating the detuning of pump laser 1.

\section{Bandwidth of photon pair versus cell temperature}

Because of the Doppler effect, the bandwidth of the generated photon is broadened. The bandwidth of the generated photon pair is therefore different at different $\mathrm{Rb}$ cell temperatures. We measure the time-width of the coincidence window versus the cell temperature and obtain the relationship between the time-width of the coincidence and temperature. The time-width of the generated photon becomes narrower with increasing temperature in the atomic cell. The recorded results are shown in Fig. S2.

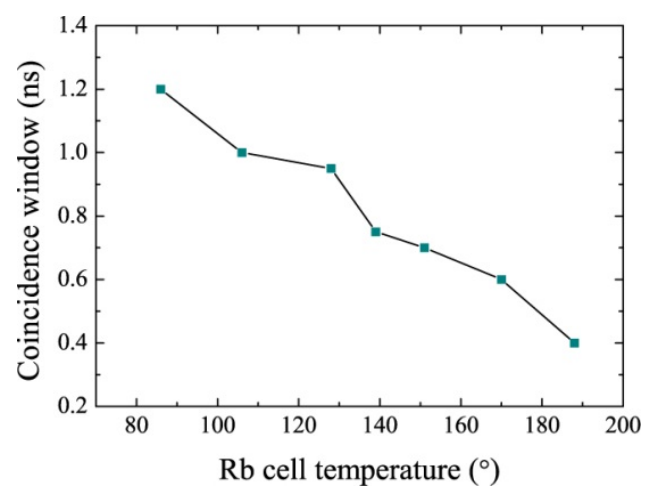

Fig. S2. Measured coincidence window of the signal 1 and signal 2 photons versus cell temperature. In this case, the coincidence window represents the time width of the entire coincidene wave packet. 
Proof of extended Cauchy-Schwarz inequality for three variables

We define three unrelated random variables $a_{i}, b_{i}, c_{i}$ $(i=0 \sim \mathrm{m})$. According to the Cauchy-Schwarz inequality for two variables:

$$
\left(\sum_{i=1}^{m} a_{i} b_{i}\right)^{2} \leq \sum_{i=1}^{m} a_{i}^{2} \sum_{i=1}^{m} b_{i}^{2}
$$

By considering $a_{i} b_{i}$ as a variable, we then obtain:

$$
\left(\sum_{i=1}^{m} a_{i} b_{i} c_{i}\right)^{2} \leq \sum_{i=1}^{m} a_{i}^{2} b_{i}^{2} \sum_{i=1}^{m} c_{i}^{2}
$$

Because $\sum_{i=1}^{m} a_{i}^{2} b_{i}^{2} \leq \sum_{i=1}^{m} a_{i}^{2} \sum_{i=1}^{m} b_{i}^{2}$, the final inequality thus can be found as follows:

$$
\left(\sum_{i=1}^{m} a_{i} b_{i} c_{i}\right)^{2} \leq \sum_{i=1}^{m} a_{i}^{2} b_{i}^{2} \sum_{i=1}^{m} c_{i}^{2} \leq \sum_{i=1}^{m} a_{i}^{2} \sum_{i=1}^{m} b_{i}^{2} \sum_{i=1}^{m} c_{i}^{2} .
$$

The extended Cauchy-Schwarz inequality for three variables can thus be written as:

$$
\langle u v \kappa\rangle^{2} \leq\left\langle u^{2}\right\rangle\left\langle v^{2}\right\rangle\left\langle\kappa^{2}\right\rangle
$$

where $u, v, \kappa$ are unrelated random variables.

Estimation of the auto-correlation of the signal 3 and signal 4 photons

We input a weak coherent laser beam at $780 \mathrm{~nm}$ into the PPLN waveguide to generate and then measure the auto-correlation of the signal 3 and signal 4 photons for different 780-nm laser input powers. The measured results are shown in Fig. S3. With decreasing 780-nm laser power, the probability of multi-photons from signal 3 and signal 4 decreases. Based on this process, the auto-correlation of signal 3 and signal 4 is estimated to be $g_{s 3, s 3}^{(2)}(0)=g_{s 4, s 4}^{(2)}(0)=1 \pm 0.05$.

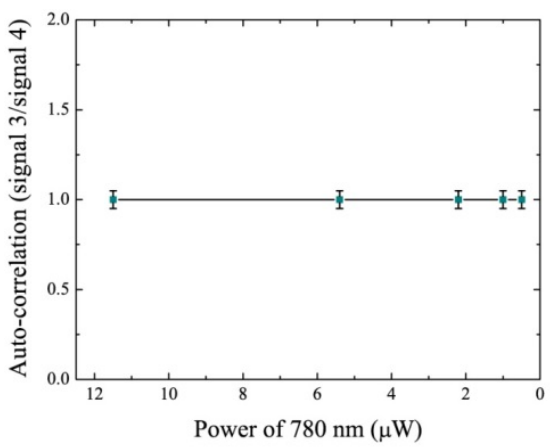

Fig. S3. Measured auto-correlation of signal 3 and signal 4 photons versus the power of the 780-nm laser. The error bars indicate the experimental statistics.

\section{REFERENCES}

1. Ding Dong-Sheng, Zhou Zhi-Yuan, Shi Bao-Sen, "Slow Light via FourWave Mixing in a Hot Rubidium Vapor". Chin. Phys. B 22, 114203 (2013). 